\title{
Spectrophotometric Analysis of Different Flowable Restorative Materials
}

\author{
Abeer E ElEmbaby ${ }^{1}$, Mouneir Slais ${ }^{2}$, Ali Alawami ${ }^{3}$, Mohammed Alabbad ${ }^{4}$, Mishari Alsowyan ${ }^{5}$, Neveen Hanna ${ }^{6}$, Hala Bahgat $^{7}$
}

\begin{abstract}
Aim: To compare the color change of three flowable composites after exposure to staining solutions.

Materials and methods: A total of 240 oval samples were constructed of conventional flowable resin composite, self-adhesive flowable resin composite, and nanohybrid flowable ormocer $(n=80)$. Each set was further subcategorized into three subsets as regards staining solution. The baseline color parameters were recorded using spectrophotometer after the immersion of the specimens in artificial saliva for 24 hours. Then, the measurements were taken again after exposure to staining solutions where the color change was measured as $\Delta E^{*}=\sqrt{ }\left(\Delta L^{* 2}+\Delta \mathrm{a}^{* 2}+\Delta \mathrm{b}^{* 2}\right)$. Results: Conventional flowable resin composite had the best color stability in all staining solutions. The coffee was concluded to be the most staining solution. Compared to beverages, mouthwashes had a greater discoloration effect on nanohybrid flowable ormocer than on selfadhesive flowable composite.

Conclusion: Conventional flowable composite had the highest color stability among the tested materials, followed by flowable nanohybrid ormocer, and the least was self-adhering flowable composite. Coffee had the greatest discoloration effect, followed by a sports drink and then the energy drink. However, mouthwashes did not have a significant discoloration effect.

Clinical significance: Selection of flowable composite materials that are resistant to staining by coffee, beverages, or mouthwashes is an important factor that may influence the sustainability of the esthetic quality of the restoration.

Keywords: Color change, Flowable composites, Staining solutions.

The Journal of Contemporary Dental Practice (2021): 10.5005/jp-journals-10024-3031
\end{abstract}

\section{INTRODUCTION}

The growing esthetic expectations and the demands of dental patients resulted in the widespread use of composite restorative materials. ${ }^{1,2}$ Flowable composites were first introduced in the year 1995. They have superior esthetic properties, low viscosity, and excellent handling characteristics. However, the flowable composites do not bond with the tooth structure; thus, the use of bonding agents is necessary. Nowadays, self-adhering flowable composite has been introduced to overcome multiple, techniquesensitive, and longer-duration procedures of conventional bond. ${ }^{3-5}$

It is designed to interact micromechanically and chemically with the tooth, by the inclusion of an acidic adhesive monomer, namely glycerophosphate dimethacrylate (GPDM), in their composition. ${ }^{6-8}$

Organically modified ceramic (ormocer) technology, established by the German-based, Fraunhofer Institute for Silicate Research, has been implemented by many industries for its beneficial optical properties, scratch resistance, and antimicrobial behaviors. Admira Fusion Flow is the worldwide first purely ormocer-based flowable composite. Its chief advantage is the lower polymerization contraction ( $2.75 \%$ by volume) compared to the traditional flowable resins. ${ }^{9}$

Successful esthetic restoration is a multifactorial achievement, where the simulation of tooth color as well as the stability of this color during service in the harsh oral environment is crucial. ${ }^{10}$ Because of the relative novelty of both self-bonding and ormocerbased flowable composites, it seemed interesting to investigate further on their color stability; hence, the goal of this laboratory research was to assess their color changes spectrophotometrically after exposure to solutions, in comparison with the conventional flowable composite. The tested null hypothesis was that statistically
1,6,7 Department of Restorative Dental Sciences, College of Dentistry, Imam Abdulrahman Bin Faisal University, Dammam, Kingdom of Saudi Arabia

${ }^{2-5}$ Ministry of Health, Riyadh, Eastern Region, Kingdom of Saudi Arabia Corresponding Author: Abeer E ElEmbaby, Department of Restorative Dental Sciences, College of Dentistry, Imam Abdulrahman Bin Faisal University, Dammam, Saudi Arabia, Phone: +966 38574928, e-mail: aeelembaby@iau.edu.sa

How to cite this article: ElEmbaby $A E$, Slais $M$, Alawami $A$, et al. Spectrophotometric Analysis of Different Flowable Restorative Materials. J Contemp Dent Pract 2021;22(2):111-116.

Source of support: Nil

Conflict of interest: None

insignificant color changes are achieved by the conventional, selfadhering, and ormocer-based flowable composites.

\section{Materials and Methods}

The laboratory investigation was performed throughout 6 months in the college of Dentistry Materials Research Laboratory, Imam Abdulrahman University, KSA.

\section{Grouping of the Specimens}

A total of 240 samples were categorized into three sets $(n=80)$ as regards the restorative materials: Filtek Z350 XT Flowable (FZX conventional light-cured flowable resin composite; 3M ESPE, St Paul, Minnesota, USA), Fusio Liquid Dentin (FLD self-adhering flowable resin composite; Pentron Clinical), and Admira Fusion Flow (AFF flowable light-curing nanohybrid ormocer; $\mathrm{VOCO}, \mathrm{GmbH}$, Germany). 
Further subgrouping is shown in the flowchart (Fig. 1) and classified as follows:

Groups A, B, and C indicate the type of flowable composites used as follows:

- Group A: Filtek Z350 XT Flowable $(n=80)$

- Group B: Fusio Liquid Dentin $(n=80)$

- Group C: Admira Fusion Flow $(n=80)$

Subgroups 1, 2, and 3 and sub-subgroups I to VII specify immersion solution type as follows:

- Subgroup 1: Freshly prepared artificial saliva (control) $(n=10)$

- Subgroup 2: Mouthwashes (MW)

o Sub-subgroup I: Avohex (chlorhexidine MW), Middle East Pharmaceutical Industries, pH $5.8(n=10)$

o Sub-subgroup II: Colgate Plax (fluoride-containing MW), Colgate Palmolive, pH $6.1(n=10)$

o Sub-subgroup III: Crest 3D white (bleaching MW), Procter and Gamble Manufacturing Company, pH $5.4(n=10)$

o Sub-subgroup IV: Listerine original (alcohol-containing MW), Johnson and Johnson Limited, $\mathrm{pH} 4.2(n=10)$

- Subgroup 3: Beverages

o Sub-subgroup V: Coffee, Folgers Coffee J.M. Smucker Company, pH $5.2(n=10)$

o Sub-subgroup VI: Sports drink, The Gatorade, PepsiCo, $\mathrm{pH} 3.1(n=10)$

o Sub-subgroup VII: Energy drink, Power Horse, S. Spitz GmbH, $\mathrm{pH} 3(n=10)$

\section{Specimens' Preparation}

The oval disk-shaped specimens (diameter, $12 \times 10 \mathrm{~mm}$ and thickness, $2 \mathrm{~mm}$ ) were fabricated according to ISO 7491:2000. ${ }^{11}$ Restorative materials were prepared using a Teflon mold as described in detail in previous studies. ${ }^{12-14}$ The samples were bathed in $20 \mathrm{~mL}$ of the immersing medium within an opaque flask kept in an incubator (JRAD, China) at $37^{\circ} \mathrm{C}$ for 24 hours for artificial saliva and mouthwash solutions and 2 weeks for beverage solutions. ${ }^{15}$ The color changes $(\Delta E)$ were obtained using the procedure given in a previous study, ${ }^{16}$ and the formula is given as

$$
\Delta E^{*}=\sqrt{\left(\Delta L^{* 2}+\Delta a^{* 2}+\Delta b^{* 2}\right)}
$$

where $L^{*}$ coordinate represents the color luminosity, and $a^{*}$ and $b^{*}$ coordinates represent the color chromaticity. ${ }^{17,18} \Delta E$ was considered not detectable when it was equal to or less than 1 unit, detectable between 1 and 3.3 units, and not accepted clinically when it was more than 3.3 units. $^{19}$

\section{Statistical Analysis}

Data were statistically analyzed with a repeated measures analysis of variance (ANOVA) and Tukey's test ( $p<0.05$ ) through SPSS 20.0 (IBM Product, Chicago, USA). One-way ANOVA was conducted to check the effects in terms of $\Delta L, \Delta A, \Delta B$, and $\Delta E$ of various solutions in comparison to artificial saliva (control), and among the three restorative materials (i.e., FZX, FLD, and AFF). Repeated measures ANOVA with four factors was used to compare the effects of $\Delta L$, $\Delta A, \Delta B$, and $\Delta E$ within each solution.

\section{Results}

FZX had the best color stability in solutions, where the $\Delta E$ for the FZX had no visible difference in each tested solution except for coffee. Meanwhile, all mouthwashes had a greater discoloration effect on AFF than on FLD, and all the beverages and artificial saliva had a greater discoloration effect on FLD than on AFF material except for coffee. Tables 1 to 5 and Figures 1 to 3 illustrate the effect of color stability on restorative materials.

Also, there was no significant relation between discoloration and the $\mathrm{pH}$ of the staining solutions. For AFF with every type of staining solutions, $\Delta E$ had a perceptible difference; however, it was clinically not an acceptable difference in coffee solution.

On the other hand, FLD had no visible difference in bleaching MW, fluoride MW, and alcoholic MW; perceptible difference in chlorhexidine MW; and finally clinically unacceptable for coffee, sports drink, and energy drink.

\section{Discussion}

The long-term color stability of esthetic restorative materials is considered a crucial property influencing their success during the oral service. In the present study, commonly used beverages and mouthwashes were used to immerse the specimens for 24 hours and 2 weeks, respectively, which is considered enough for the assessment of staining vulnerability. ${ }^{20}$

The color shift of esthetic restorative materials during their service is considered a multifaceted problem that can be classified as follows: (1) exogenous staining as a sequence of plaque and stain precipitation, (2) surface deterioration due to absorption of colorants onto the resin, and (3) endogenous discoloration caused by physicochemical reactions in the body of the material. This

Table 1: $\Delta E$ of different staining solutions on ormocer nanohybrid

\begin{tabular}{|c|c|c|c|c|c|c|}
\hline \multirow[b]{2}{*}{ Solution } & \multicolumn{2}{|r|}{ Admira fusion flow } & \multicolumn{2}{|r|}{ Fusio liquid dentin } & \multicolumn{2}{|c|}{ Filtek Z350 XT flowable } \\
\hline & $\Delta E$ & Evaluation & $\Delta E$ & Evaluation & $\Delta E$ & Evaluation \\
\hline Chlorhexidine MW & 1.24 & Perceptible D & 1.10 & Perceptible D & 0.61 & Nonvisible D \\
\hline Fluoride MW & 1.36 & Perceptible D & 0.89 & Nonvisible D & 0.91 & Nonvisible D \\
\hline Bleaching MW & 1.30 & Perceptible D & 0.58 & Nonvisible D & 0.85 & Nonvisible D \\
\hline Alcoholic MW & 1.35 & Perceptible D & 0.68 & Nonvisible D & 0.63 & Nonvisible D \\
\hline Coffee & 8.82 & Clinical unacceptable D & 7.85 & Clinical unacceptable D & 6.30 & Clinical unacceptable D \\
\hline Sports drink & 1.91 & Perceptible D & 3.34 & Clinical unacceptable D & 0.56 & Nonvisible D \\
\hline Energy drink & 1.06 & Perceptible D & 3.54 & Clinical unacceptable D & 0.68 & Nonvisible D \\
\hline Artificial saliva & 1.19 & Perceptible D & 1.34 & Perceptible D & 0.28 & Nonvisible D \\
\hline
\end{tabular}


Spectrophotometry of Flowable Restorative Materials

Table 2: Effect of various solutions on color change in Admira fusion flow

\begin{tabular}{|c|c|c|c|c|c|}
\hline \multirow[b]{2}{*}{ Solutions } & \multicolumn{4}{|c|}{ Color change } & \multirow[b]{2}{*}{ Pvalue } \\
\hline & $\Delta L$ & $\Delta A$ & $\Delta B$ & $\Delta E$ & \\
\hline A1 (chlorhexidine MW) & $0.348 \pm 0.150$ & $0.122 \pm 0.047$ & $1.170 \pm 0.410$ & $1.249 \pm 0.354$ & 0.002 \\
\hline A2 (fluoride MW) & $0.548 \pm 0.217$ & $0.180 \pm 0.178$ & $1.156 \pm 0.953$ & $1.360 \pm 0.872$ & 0.004 \\
\hline A3 (bleaching MW) & $0.610 \pm 0.268$ & $0.126 \pm 0.047$ & $1.074 \pm 0.851$ & $1.309 \pm 0.764$ & 0.003 \\
\hline A4 (alcoholic MW) & $1.000 \pm 0.477$ & $0.074 \pm 0.031$ & $0.736 \pm 0.401$ & $1.350 \pm 0.209$ & 0.004 \\
\hline B1 (coffee) & $4.442 \pm 0.756^{*}$ & $0.878 \pm 0.066^{*}$ & $7.556 \pm 0.104^{*}$ & $8.825 \pm 0.483^{*}$ & 0.002 \\
\hline B2 (sports drink) & $0.456 \pm 0.465$ & $0.292 \pm 0.078^{*}$ & $1.804 \pm 0.723$ & $1.909 \pm 0.789$ & 0.004 \\
\hline B3 (energy drink) & $0.612 \pm 0.268$ & $0.154 \pm 0.086$ & $0.764 \pm 0.507$ & $1.063 \pm 0.389$ & 0.008 \\
\hline C1 (artificial saliva) & $0.590 \pm 0.428$ & $0.080 \pm 0.042$ & $0.978 \pm 0.278$ & $1.190 \pm 0.363$ & 0.004 \\
\hline$P$ value & 0.001 & 0.001 & 0.001 & 0.001 & \\
\hline
\end{tabular}

*Significant color change under various solutions as compared to control C1 (artificial saliva)

Table 3: Effect of various solutions on color change in Filtek Z350 XT Flowable

\begin{tabular}{lllll}
\hline & \multicolumn{4}{c}{ Color change } \\
\cline { 2 - 5 } Solutions & $\Delta L$ & $\Delta A$ & $\Delta B$ & $\Delta E$ \\
\hline A1 (chlorhexidine MW) & $0.496 \pm 0.135$ & $0.152 \pm 0.048$ & $0.292 \pm 0.239$ & $0.617 \pm 0.211$ \\
A2 (fluoride MW) & $0.766 \pm 0.107$ & $0.088 \pm 0.059$ & $0.416 \pm 0.325$ & $0.920 \pm 0.149$ \\
A3 (bleaching MW) & $0.704 \pm 0.219$ & $0.144 \pm 0.073$ & $0.422 \pm 0.190$ & $0.857 \pm 0.197$ \\
A4 (alcoholic MW) & $0.534 \pm 0.378$ & $0.082 \pm 0.036$ & $0.260 \pm 0.195$ & $0.633 \pm 0.362$ \\
B1 (coffee) & $2.572 \pm 0.765^{*}$ & $0.128 \pm 0.100$ & $5.752 \pm 2.009^{*}$ & $6.307 \pm 2.135^{*}$ \\
B2 (sports drink) & $0.416 \pm 0.169$ & $0.284 \pm 0.061^{*}$ & $0.206 \pm 0.100$ & $0.565 \pm 0.115$ \\
B3 (energy drink) & $0.406 \pm 0.177$ & $0.174 \pm 0.072$ & $0.452 \pm 0.260$ & 0.003 \\
C1 (artificial Saliva) & $0.232 \pm 0.255$ & $0.054 \pm 0.025$ & $0.116 \pm 0.222$ & 0.003 \\
$P$ value & 0.001 & 0.001 & 0.001 & 0.011 \\
\hline
\end{tabular}

*Significant color change under various solutions as compared to control C1 (artificial saliva)

Table 4: Effect of various solutions on color change in Fusio liquid dentin

\begin{tabular}{|c|c|c|c|c|c|}
\hline \multirow[b]{2}{*}{ Solutions } & \multicolumn{4}{|c|}{ Color change } & \multirow[b]{2}{*}{ Pvalue } \\
\hline & $\Delta L$ & $\Delta A$ & $\Delta B$ & $\Delta E$ & \\
\hline A1 (chlorhexidine MW) & $0.908 \pm 0.363$ & $0.098 \pm 0.083$ & $0.512 \pm 0.423$ & $1.110 \pm 0.386$ & 0.005 \\
\hline A2 (fluoride MW) & $0.762 \pm 0.216$ & $0.254 \pm 0.186$ & $0.236 \pm 0.270$ & $0.897 \pm 0.161$ & 0.013 \\
\hline A3 (bleaching MW) & $0.320 \pm 0.263$ & $0.100 \pm 0.039$ & $0.412 \pm 0.293$ & $0.581 \pm 0.296$ & 0.014 \\
\hline A4 (alcoholic MW) & $0.420 \pm 0.504$ & $0.090 \pm 0.037$ & $0.426 \pm 0.542$ & $0.683 \pm 0.652$ & 0.017 \\
\hline B1 (coffee) & $2.942 \pm 0.618^{*}$ & $1.322 \pm 0.428^{*}$ & $6.646 \pm 3.766^{*}$ & $7.848 \pm 2.443^{*}$ & 0.007 \\
\hline B2 (sports drink) & $1.064 \pm 0.793$ & $0.680 \pm 0.326^{*}$ & $3.024 \pm 1.011$ & $3.341 \pm 1.109$ & 0.004 \\
\hline B3 (energy drink) & $1.376 \pm 1.186$ & $0.316 \pm 0.124$ & $3.156 \pm 2.064$ & $3.546 \pm 2.215$ & 0.003 \\
\hline C1 (artificial saliva) & $0.270 \pm 0.260$ & $0.124 \pm 0.098$ & $1.208 \pm 1.071$ & $1.346 \pm 0.942$ & 0.019 \\
\hline$P$ value & 0.001 & 0.001 & 0.001 & 0.001 & \\
\hline
\end{tabular}

*Significant color change under various solutions as compared to control C1 (artificial saliva)

Table 5: Comparison of color changes among the three groups

\begin{tabular}{lllll}
\hline & \multicolumn{3}{c}{ Groups } & \\
\cline { 2 - 4 } Color change & Admira & Filtik 3M & Fusio & Pvalue \\
\hline$\Delta L$ & $1.076 \pm 1.355$ & $0.766 \pm 0.774$ & $1.008 \pm 0.994$ & 0.397 \\
$\Delta A$ & $0.238 \pm 0.265$ & $0.138 \pm 0.089$ & $0.373 \pm 0.452^{*}$ & $0.004^{*}$ \\
$\Delta B$ & $1.905 \pm 2.251$ & $0.990 \pm 1.946$ & $1.953 \pm 2.580$ & 0.107 \\
$\Delta E$ & $2.282 \pm 2.569$ & $1.358 \pm 2.032$ & $2.419 \pm 2.634$ & 0.109 \\
\hline
\end{tabular}

*Significant at $5 \%$ level of significance color shift has been attributed to composite resin components, mainly the monomer matrix and the filler particles, as proved in other studies. ${ }^{21,22}$

The findings of this study displayed that the FZX had the best color stability in all different solutions. This could be attributed mainly to its filler component which is a blend of nonagglomerated 20-nm silica and 4- to 11-nm zirconia fillers, and silica/zirconia filler clusters, thus producing a wide variety of sizes allowing a relatively increased filler percentage. This is reflected in the better color 


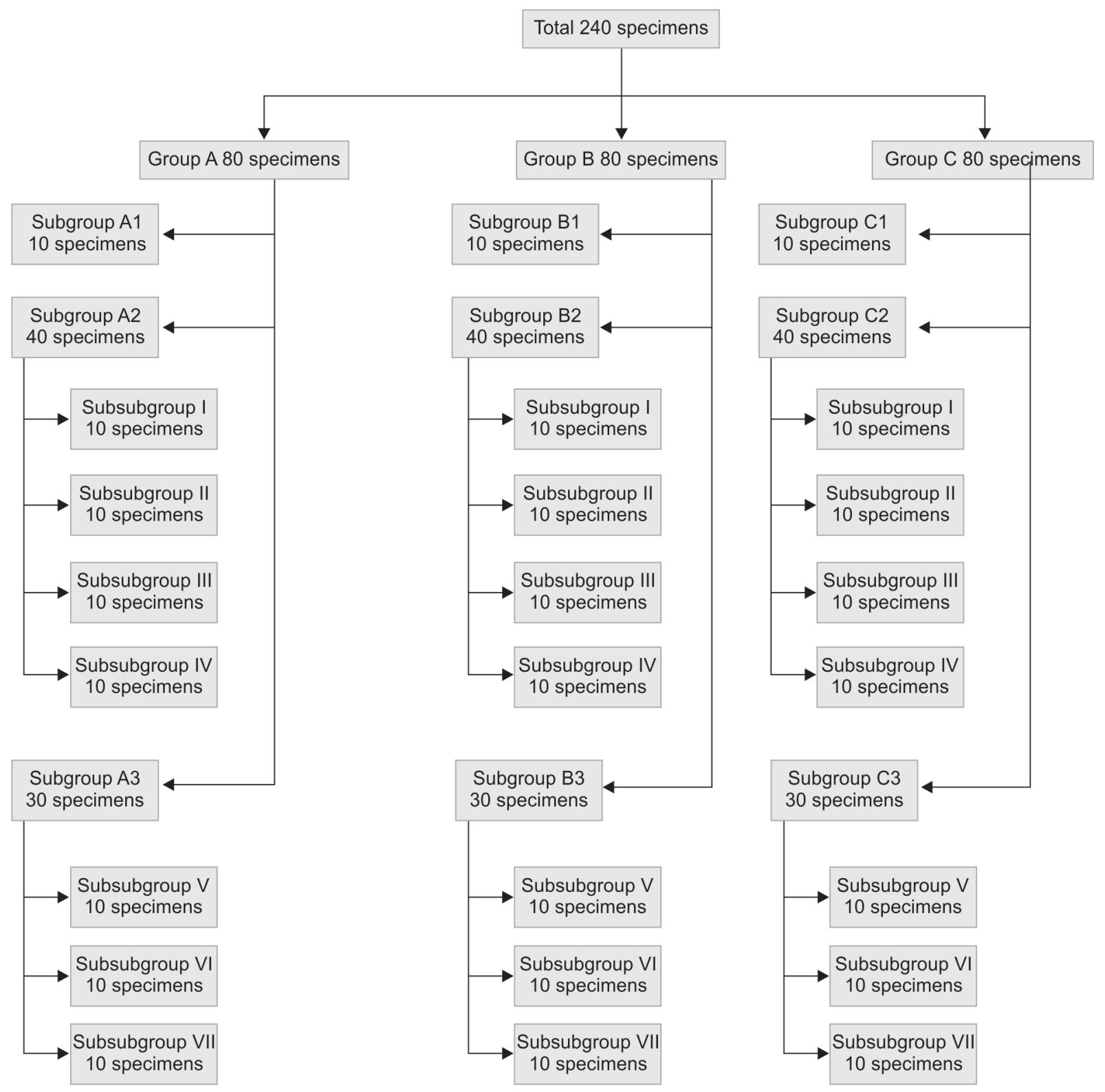

Flowchart: Flowchart showing grouping and subgrouping of samples

stability of FZX when immersed in the investigated solutions, as high filler loading is always associated with better properties of composite resin materials. ${ }^{23,24}$ Also, this proved the color stability of FZX agrees with another investigation which documented that incorporation of finer particles into resin composite led to less interparticle space and safeguarded the more susceptible and softer resin matrix. ${ }^{25}$ This consequently generated a smooth surface texture, which was resistant to staining by different solutions.

Another important factor which also impacted the color stability of the investigated composites is the degree of conversion of their resin matrices. Generally, a high degree of $\mathrm{C}=\mathrm{C}$ conversion may impact color steadiness, as the remaining monomer in the organic matrix may result in colorimetric degradation products that facilitate the diffusion of solvents from the oral cavity into the organic matrix. ${ }^{26}$ This concept might explain the findings of the current study that all investigated mouthwashes had a greater discoloration effect on AFF than on FLD composite, where the lower double-bond conversion of the matrix of AFF could be responsible for this pitfall, when compared to dimethacrylate-based composite, as ormocer-based composite is renowned as highly functionalized compounds, thus supporting denser networks, where the double bonds are less accessible to polymerization, leaving a large fraction of unreacted double bond behind. Such an inadequate polymerized matrix is usually vulnerable to discoloration. ${ }^{27}$

All beverages and artificial saliva had a greater discoloration effect on FLD than on AFF restorative material. This may be related to the hydrophilic behavior of the organic matrix of FLD due to the presence of hydroxyethyl methacrylate (HEMA), which could lead to a significant increase in the water uptake with subsequent degradation and color shift. ${ }^{28}$

The coffee is the only solution which strongly affected all materials when compared to other solutions in this study, and it is due to its high affinity to the polymer network. ${ }^{29-31}$ Interestingly, 


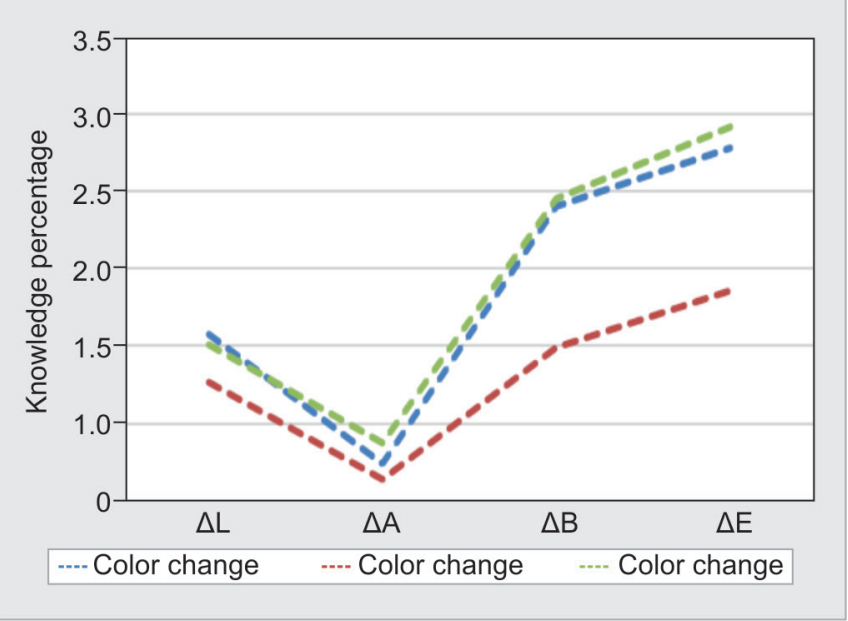

Fig. 2: A graph showing the comparison between the color changes of tested restorative materials

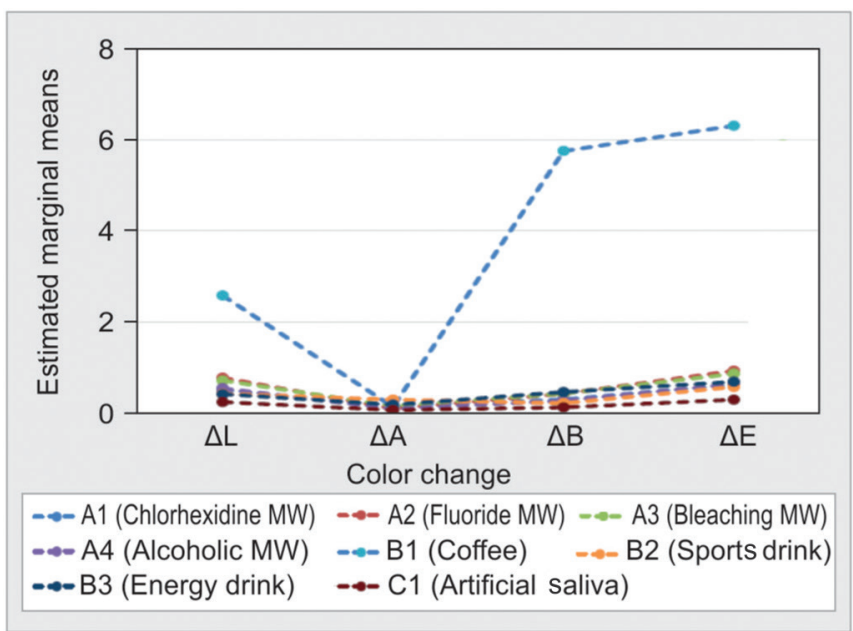

Fig. 3: A graph showing the comparison of color changes with different tested solutions

and in agreement to previous study, there was no relation between discoloration and $\mathrm{pH}$ of the immersed investigated solutions. ${ }^{32}$

\section{LiMitaTiONS}

The information about the ingredient ratio of the restorative material composition is not available like a trade secret from the manufacturer.

\section{Conclusions}

Based on our findings, the following can be concluded:

- The highest color stability among the tested materials was for conventional flowable resin composite, followed by flowable light-curing nanohybrid ormocer restorative, and the least was for self-adhering flowable resin composite.

- Coffee had the greatest discoloration effects, followed by sports drink and then an energy drink. Artificial saliva and mouthwashes had similar values of discoloration effect.

\section{References}

1. Arregui $M$, Giner L, Ferrari $M$, et al. Six-month color change and water sorption of nine new-generation flowable composites in 6 staining solutions. Braz Oral Res 2016;30(1):e123. DOI: 10.1590/1807-3107BOR2016.vol30.0123.

2. Miyagawa Y, Powers JM, O'Brien WJ. Optical properties of direct restorative materials. J Dent Res 1981;60:890-894. DOI: $10.1177 / 00220345810600050601$

3. Hegde MN, Hegde P, Chandra CR. Morphological evaluation of new total etching and self-etching adhesive system interfaces with dentin. J Conserv Dent 2012;15(2):151-155. DOI: 10.4103/0972-0707.94589.

4. Eliades $G$, Vougiouklakis $G$, Palaghias $G$. Heterogeneous distribution of single-bottle adhesive monomers in the resin-dentin interdiffusion zone. Dent Mater 2001;17(4):277-283. DOI: 10.1016/s01095641(00)00082-8.

5. Koliniotou-Koumpia E, Kouros P, Koumpia E, et al. Shear bond strength of a "solvent-free" adhesive versus contemporary adhesive systems. Braz J Oral Sci 2014;13(1):64-69. DOI: 10.1590/1677-3225v13n1a13.

6. Sadeghi M. An in vitro microleakage study of Class V cavities restored with a new self-adhesive flowable composite resin versus different flowable materials. Dent Res J (Isfahan) 2012;9(4):460-465. DOI: 10.4103/1735-3327.102784.

7. Bektas OO, Eren D, Akin EG, et al. Evaluation of a self-adhering flowable composite in terms of micro-shear bond strength and micro leakage. Acta Odontol Scand 2013;71(3-4):541-546. DOI: 10.3109/00016357.2012.696697.

8. Vichi A, Margvelashvili M, Goracci C, et al. Bonding and sealing ability of a new self-adhering flowable composite resin in Class I restorations. Clin Oral Investig 2013;17(6):1497-1506. DOI: 10.1007/s00784-012-0846-6.

9. Llena C, Fernández S, Forner L. Color stability of nanohybrid resinbased composites, ormocers and compomers. Clin Oral Investig 2017;21:1071-1077. DOI: 10.1007/s00784-016-1850-z.

10. ElEmbaby AE-S. The effects of mouth rinses on the color stability of resin-based restorative materials. J Esthet Restor Dent 2014;26(4):264271. DOI: 10.1111/jerd.12061.

11. Gürdal P, Güniz B, Akdeniz, et al. The effects of mouth rinses on microhardness and color. J Oral Rehabil 2002;29:895-901. DOI: 10.1046/j.1365-2842.2002.00924.x.

12. Poggio C, Ceci M, Beltrami R, et al. Color stability of esthetic restorative materials: a spectrophotometric analysis. Acta Biomater Odontol Scand 2016;2(1):95-101. DOI: 10.1080/23337931.2016.1217416.

13. Lang NP, Tan WC, Krahenmann MA, et al. A systematic review product of the effects of full-mouth debridement with and without antiseptics in patients with chronic periodontitis. J Clin Periodontol 2008;35:8-21. DOI: 10.1111/j.1600-051X.2008.01257.x.

14. Fischman SL. A clinician's perspective on antimicrobial mouth rinses. Am Dent Assoc 1994;125:20-22. DOI: 10.1016/s0002-8177(94)14003-3.

15. Yazici AR, Celik C, Dayangac B, et al. The effect of curing units and staining solutions on the color stability of resin composites. Oper Dent 2007;32:616-622. DOI: 10.2341/07-3.

16. Celik C, Yuzugullu B, Erkut S, et al. Effects of mouth rinses on color stability of resin composites. Eur J Dent 2008;2:247-253. DOI: 10.1055/s-0039-1697388.

17. Nasim I, Neelakantan P, Sujeer R, et al. Color stability of microfilled, microhybrid and nanocomposite resins-An in vitro study. J Dent 2010;38:137-142. DOI: 10.1016/j.jdent.2010.05.020.

18. Mundim FM, Garcia LDFR, Pires-De-Souza FDCP. Effect of staining solutions and repolishing on color stability of direct composites. J Appl Oral Sci 2010;18(3):249-254. DOI: 10.1590/s167877572010000300009.

19. Bagheri R, Burrow $M$, Tyas $M$. Influence of food-simulating solutions and surface finish on susceptibility to staining of aesthetic restorative materials. J Dent 2005;33(5):389-398. DOI: 10.1016/j. jdent.2004.10.018

20. Ertas E, Güler AU, Yücel AC, et al. Color stability of resin composites after immersion in different drinks. Dent Mater J 2006;25:371-376. DOI: $10.4012 / \mathrm{dmj} .25 .371$. 
21. Dietschi D, Campanile G, Holz J, et al. Comparison of the color stability of ten new-generation composites: an in vitro study. Dent Mater 1994;10:353-362. DOI: 10.1016/0109-5641(94)90059-0.

22. Lee YK, Powers JM. Discoloration of dental resin composites after immersion in a series of organic and chemical solutions. J Biomed Mater Res B Appl Biomater 2005;73:361-367. DOI: 10.1002/ jbm.b.30216.

23. Van Landuyt KL, Snauwaert J, De Munck J, et al. Systematic review of the chemical composition of contemporary dental adhesives. Biomaterials 2007;28:3757-3785. DOI: 10.1016/j. biomaterials.2007.04.044.

24. Reis AF, Giannini M, Lovadino JR, et al. The effect of six polishing systems on the surface roughness of two packable resin-based composites. Am J Dent 2002;15:193-197. PMID: 12469758.

25. Alzraikat H, Burrow MF, Maghaireh GA, et al. Nanofilled resin composite properties and clinical performance. A review. Oper Dent 2018;43:173-190. DOI: 10.2341/17-208-T.

26. Kheraif AA. Effects of curing units and the staining solutions on the color susceptibility of a microhybrid composite resin. J Dent Sci 2011;6:33-40. DOI: 10.1016/j.jds.2011.02.006

27. Moszner N, Gianasmidis A, Klapdohr S, et al. Sol-gel materials 2. Light-curing dental composites based on ormocers of cross-linking alkoxysilane methacrylates and further nanocomponents. Dent Mater 2008;24:851-856. DOI: 10.1016/j.dental.2007.10.004.

28. Sideridou I, Tserki V, Papanastasiou G. Study of water sorption, solubility and modulus of elasticity of light-cured dimethacrylatebased dental resins. Biomaterials 2003;24:655-665. DOI: 10.1016/ s0142-9612(02)00380-0.

29. Kalra S, Singh A, Gupta M, et al. Ormocer: an aesthetic direct restorative material; an in vitro study comparing the marginal sealing ability of organically modified ceramics and a hybrid composite using an ormocer-based bonding agent and a conventional fifthgeneration bonding agent. Contemp Clin Dent 2012;3:48-53. DOI: 10.4103/0976-237X.94546.

30. Malhotra N, Shenoy RP, Acharya S, et al. Effect of three indigenous food stains on resin-based, microhybrid-, and nanocomposites. J Esthet Restor Dent 2011; 23:250-257. DOI: 10.1111/j.1708-8240.2011.00431.x.

31. Omata $Y$, Uno S, Nakaoki Y, et al. Staining of hybrid composites with coffee, oolong tea, or red wine. Dent Mater J 2006; 25:125-131. DOI: 10.4012/dmj.25.125.

32. Soares-Geraldo D, Scaramucci T, Steagall Jr W, et al. Interaction between staining and degradation of a composite resin in contact with colored foods. Braz Oral Res 2011;25:369-375. DOI: 10.1590/ s1806-83242011000400015. 